\title{
PERIOD DEPENDENT VARIATIONS OF THE SHELL SPECTRUM OF V923 AQL
}

\author{
Lubomir Iliev \\ Bulgarian Academy of Sciences \\ Institut of Astronomy \\ 72 Tsarigradsko Shosse Blvd. \\ BG-1784 Sofia, Bulgaria \\ tel./fax (+359)-2-758-927 \\ e_mail liliev@bgearn.bitnet
}

key words: Be and shell stars, binary stars

V923 Aql is a well known Be star with strong shell spectrum. It was included in the list of shell stars of Merrill and Burwell (1949). A detailed study of the radial velocity variations in the spectrum of the star based on wide collection of spectral observations was presented in the work of Koubsky et al. (1988). In this work an orbital period of 214.756 days was suggested for the binary system consisting of a B5-7 primary and low mass $(0.5 \mathrm{Msol})$.

Our study of the variations of the shell spectrum of the star is based on 10spectrograms obtained at coude-spectrograph of the $2 \mathrm{~m}$ RCC telescope at BNAO "Rozhen" in three different observing seasons. All spectrograms were digitized with Joice Loebl microdensitometer and the radial velocities were measured with the oscilloscopic comparator at BNAO "Rozhen".

Our results reveal that changes of the Balmer progression correlate with the orbital phase. Slightly positive at phase 0.0 the progression decreased gradually and at phase 0.4 is already negative and quickly decreased afterwards (fig. 1). The lack of well dispersed over the period observations can not allow us to trace the behaviour of the decrement with more details.

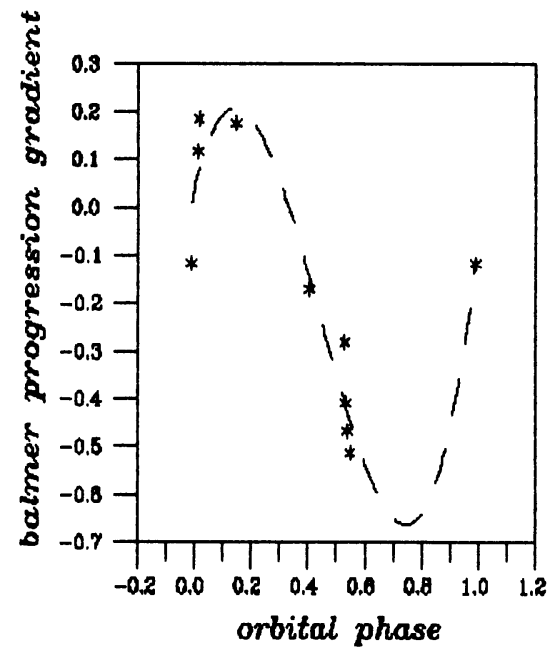

Fig. 1. Period dependent changes of the Balmer progression decrement. 


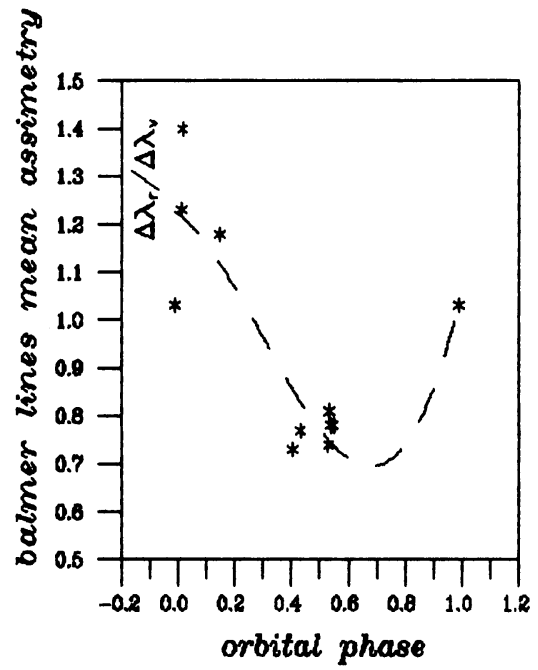

Fig. 2. Period dependent changes of the asymmetry in Balmer shell lines.

Our results are in good agreement with the conclusions of Merrill (1952) that in phases with positive radial velocities the Balmer progression is positive too. It must be specialy noted that the asimmetry of the Balmer shell lines also changes with orbital phase (fig. 2).

Our radial velocity measurements of FelI lines of multipletes 27, 37 and 38 proved the observed from Ringuelet and Sahade (1984) existence of progression in the phase of negative velocities. In all of the multipletes we observed changes of the progression connected with the orbital period following different from that of the Balmer lines behaviour. All variations observed are above the limit of accuracy. The changes of the central depths of the metal shell lines are well pronounced and also show dependance with the orbital phase.

Our results support the conclusion that shell lines of ionized metals and that of the hidrogen not only origin in different layers of the shell but this layers underwent different changes during the orbital motion.

\section{References:}

Horn J., Iliev I., Lions R.; 1989, Bull.Astron.Inst.Czechosl., vol.40, p.31.

Koubsky P., Gulliver A.F., Harmanec P., Ballereau D., Chauville J., Graf T.,

Merrill B. and Burwell C.; 1949, Astrophys.J., vol.110, p.387.

Merrill B.W.; 1952, Astrophys.J. vol.116, p.501.

Ringuelet A. and Sahade J.; 1984, Rev.Mex.Astron.Astrof., vol.6, p.208. 